\title{
Modeling Spectral Smoothness Principle for Monaural Voiced Speech Separation
}

\author{
Wei Jiang, Wenju Liu, Pengfei Hu \\ National Laboratory of Pattern Recognition (NLPR), Institute of Automation \\ Chinese Academy of Sciences, Beijing, China 100190 \\ wjiang, lwj, pfhu@nlpr.ia.ac.cn
}

\begin{abstract}
The smoothness of spectral envelope is a commonly known attribute of clean speech. In this study, this principle is modeled through oscillation degree of each time-frequency (TF) unit, and then incorporated into a computational auditory scene analysis (CASA) system for monaural voiced speech separation. Specifically, oscillation degrees of autocorrelation function (ODACF) and of envelope autocorrelation function (ODEACF) are extracted for each T-F unit, which are then utilized in TF unit labeling. Experiment results indicate that target units and interference units are distinguished more effectively by incorporating the spectral smoothness principle than by using the harmonic principle alone, and obvious segregation improvements are obtained.
\end{abstract}

\section{INTRODUCTION}

People have an outstanding ability to follow target speeches even in 'the cocktail party problem'. To imitate this ability of human's auditory systems, computational auditory scene analysis (CASA) has become an important way to perform speech segregation [1][2]. The remarkable trait of this method is that properties of noise need not to be presumed. Besides, it can be used under monaural (one microphone) condition, which can provide a lot of convenience when two or more input channels are not available.

From the perspective of grouping strategy, CASA systems can be classified into two main types, which are schema-based CASA systems and data-driven CASA systems [3][4]. As human speech analysis is primarily a bottom-up process [5], data-driven systems occupy an extremely important position in CASA, and effective features for distinguishing target units and interference units are of central concern for these systems. As fundamental frequency (F0) is a notable property of voiced speech, previous data-driven CASA systems generally perform monaural voiced speech separation based on pitch information [6]-[9]. Although considerable achievements have been obtained by these systems, the difficulties in unit labeling under low signal-to-noise ratio (SNR) still haunt researchers in this field.

Previous researchers have already made some attempts to incorporate spectral smoothness into sound separation systems

This work was supported in part by the National Nature Science Foundation of China (No.60675026, No.90820303, and No.90820011), National High Technology Development Project of China (No.20060101Z4073, No.2006AA01Z194), and the National Grand Fundamental Research 973 Program of China (No. 2004CB318105).
[10]. In this paper, the changes in oscillation degrees of (envelope) autocorrelation function waves of T-F units belonging to clean target voiced speeches are analyzed, and we found that ODACF and ODEACF change continuously when the channel number varies from zero to the maximum, though the target sounds are different. This is consistent with the spectral smoothness principle and those units with oscillation degrees deviating from this law are inclined to be intrusion units.

The rest of this paper is organized as follows. In Section 2, we make it clear that how to represent the oscillation pattern with ODACF and ODEACF. In Section 3, details of how to use these features in a CASA system are described. In Section 4, systematic evaluations of results obtained by the CASA system are given, and in Section 5 we make some conclusions.

\section{THE OSCILLATION PATTERN}

For CASA systems, an auditory front-end first transforms the input audio signal which is corrupted by interference into time-frequency domain. Specifically, this stage consists of cochlear filtering and hair-cell filtering. In this paper, cochlear filtering is executed by a gammatone filterbank with 128 channels whose center frequencies range from $50 \mathrm{~Hz}$ to 5 $\mathrm{kHz}$ in a quasi-logarithmical way and hair-cell filtering is performed using the Meddis hair-cell model [11], which aims to simulate auditory nerve transduction. The output of the Meddis model is further divided into T-F units by $20 \mathrm{~ms}$ window with $10 \mathrm{~ms}$ shift, thus a representation of the original signal called cochleagram is obtained. Each channel of the cochleagram is bandpassed from $50 \mathrm{~Hz}$ to $1050 \mathrm{~Hz}$, and the corresponding envelope of the channel response is thus obtained.

\section{A. Autocorrelation Function}

Based on the front-end processing, autocorrelation function (ACF) $A_{H}(c, m, \tau)$ and envelope autocorrelation function (EACF) $A_{E}(c, m, \tau)$ of each T-F unit $u_{c m}$ are calculated by the following formulas.

$$
A_{H}(c, m, \tau)=\frac{1}{N_{c}} \sum_{n=0}^{N_{c}-1} h(c, m T-n) h(c, m T-n-\tau)
$$


$A_{E}(c, m, \tau)=\frac{1}{N_{c}} \sum_{n=0}^{N_{c}-1} h_{E}(c, m T-n) h_{E}(c, m T-n-\tau)$

Here, delay $\tau \in[0,12.5 \mathrm{~ms}]$ with the maximum delay corresponds to $80 \mathrm{~Hz} . h(c, n)$ and $h_{E}(c, n)$ indicate hair cell output and envelope of hair cell output of channel $c$ at time step $n$ respectively. $T$ corresponds to the time shift, and $N_{c}$ is the number of samples in $20 \mathrm{~ms}$, which is the maximum delay.

\section{B. Oscillation Degree}

In this paper, we refer to oscillation degree as the level at which a wave scatters around the mean. Oscillation degrees of ACF and EACF are not only connected with the energy of each T-F unit, but also relevant to the flatness level of the wave in each unit. In fact, if a wave has large values at all time points but they nearly remain the same, the oscillation degrees also tend to be low although it carries large energy.

In fact, only if a wave is not too flat and periodical, the ODACF and ODEACF of it tend to be high. Thus, periodic waves are inclined to have high oscillation degrees of ACF or EACF if they also contain high energy. Aperiodic waves lead to flat ACF and EACF, thus their ODACF and ODEACF are low even if they oscillate fiercely themselves.

To measure the oscillation degrees of ACF and EACF mathematically, we compute the corresponding sample variances according to the following formulas, treating $\mathrm{ACF}$ and $\mathrm{EACF}$ of each T-F unit as stochastic data series.

$$
\begin{gathered}
O D A C F(c, m)=\frac{1}{N_{c}} \sum_{\tau=0}^{N_{c}}\left[A_{H}(c, m, \tau)-\bar{A}_{H}(c, m)\right]^{2} \\
O D E A C F(c, m)=\frac{1}{N_{c}} \sum_{\tau=0}^{N_{c}}\left[A_{E}(c, m, \tau)-\bar{A}_{E}(c, m)\right]^{2}
\end{gathered}
$$

Here, $\bar{A}_{H}(c, m)$ and $\bar{A}_{E}(c, m)$ are the corresponding sample mean computed by the following formulas, in which $A_{H}(c, m, \tau)$ and $A_{E}(c, m, \tau)$ are already normalized to $[0,1]$.

$$
\bar{A}_{H}(c, m)=\frac{1}{N_{c}} \sum_{\tau=0}^{N_{c}} A_{H}(c, m, \tau)
$$
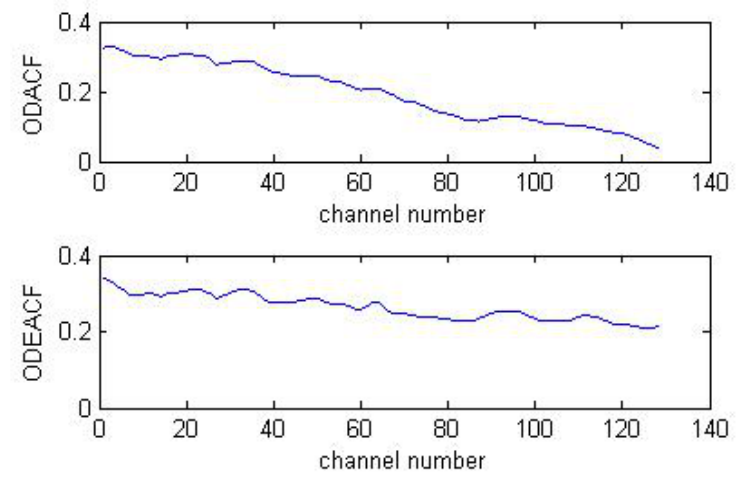

Fig. 1. Oscillation pattern of ACF and EACF for target male speeches.

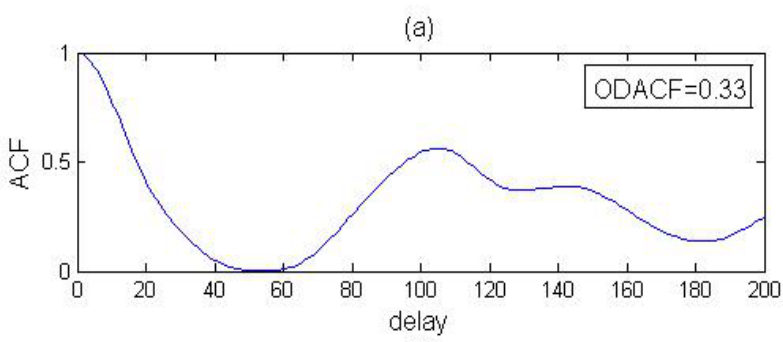

(b)

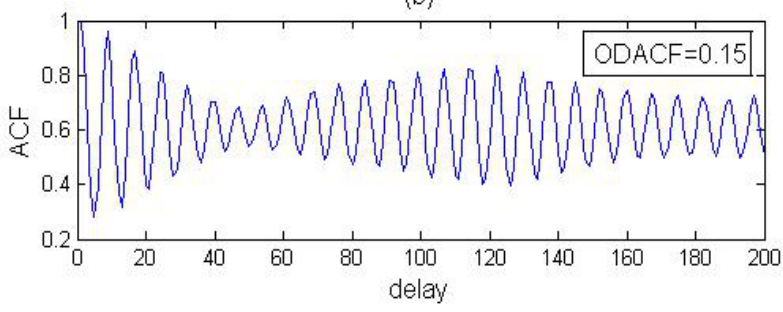

(c)

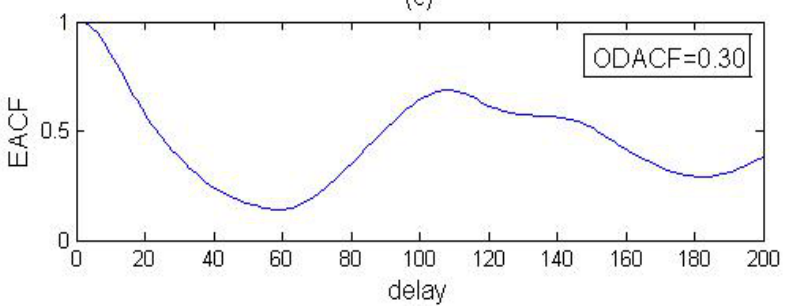

(d)

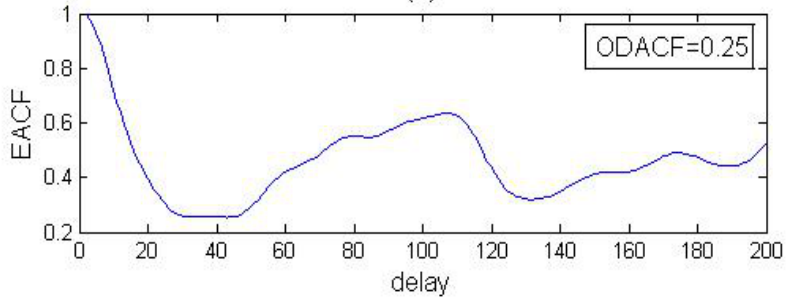

Fig. 2. (a) $A_{H}(10,90)$; (b) $A_{H}(90,90)$; (c) $A_{E}(10,90)$; (d) $A_{E}(90,90)$.

$$
\bar{A}_{E}(c, m)=\frac{1}{N_{c}} \sum_{\tau=0}^{N_{c}} A_{E}(c, m, \tau)
$$

\section{Oscillation Pattern}

As mentioned in Section I, oscillations of T-F units belonging to voiced speech signals show a consistent pattern. Specifically, we found the following facts through our experiments, where Cooke's set [6] is used as the data base.

First, oscillation degrees of ACF and EACF both decrease in a nearly linear way with the increase of the channel number. For example, these two criterions for the ten target male utterances change in a highly similar way, and the average of them is described in Figure 1. Note that there are small peaks in these two waves, and we think this is caused by the harmonic structures.

Second, oscillation degrees of EACF are larger than that of ACF in higher channels (above $1000 \mathrm{~Hz}$ ), which can also be easily seen from Figure 1. The reason for this is that amplitude modulation (beating) occurs in these unresolved channels. This phenomenon is illustrated in Figure 2. 

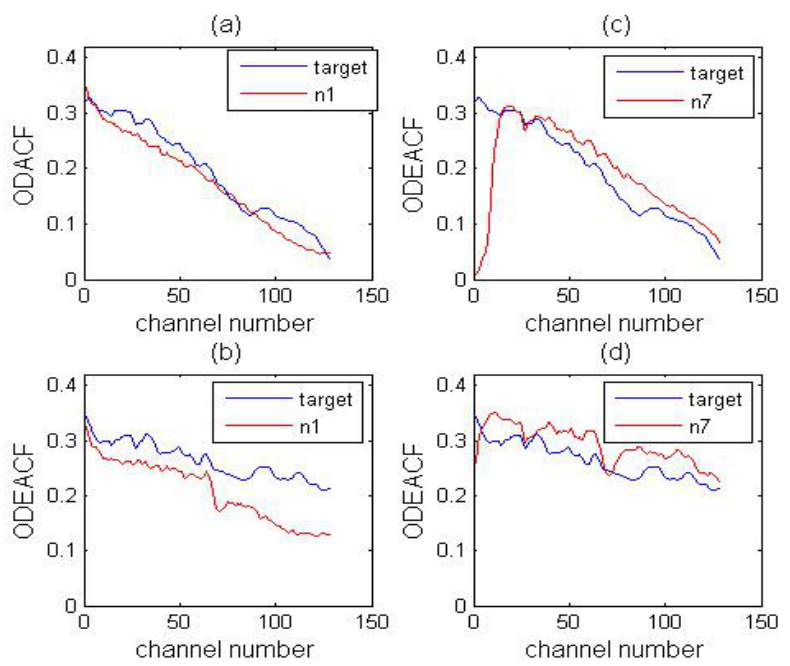

Fig. 3. Two examples deviating from the target oscillation pattern.

Third, interferences from other kind of acoustic sources usually deviate from the above two characteristics, thus could be separated from the target speeches. Two examples are depicted in Figure 3. First, ODACF and ODEACF of some intrusions are obviously lower than that of the target utterances, such as N1 (white noise) in Figure 3 (a) and (b). This happens because N1 is lack of harmonic structures and this affects a lot especially in the high channels. Second, some intrusions, such as the female utterance N7, usually have larger ODACF and ODEACF in wide spectral regions except for the lower channels, which can be seen from Figure 3 (c) and (d). This is caused by the higher pitch of N7 compared with the target speeches. Higher pitches lead to stronger energy in the high frequency regions, which makes modulation peaks and valleys in ACF and EACF waves more obvious, thus their oscillation degrees are increased.

To sum up, a uniform oscillation pattern, which is a reflection of the spectral smoothness principle, exists in the target male speeches. Meanwhile, utterances from female speakers as well as other kind of acoustic sources usually have somewhat different oscillation patterns with this. So separation of mixtures of them could be helped by oscillation degree.

\section{UTILIZING OSCILLATION PATTERN}

In this section, we illustrate how to incorporate oscillation degree of each T-F unit into an existing CASA system for monaural voiced speech segregation, and the improvements are shown in the next section.

\section{A. Pitch Computation and Grouping Strategy}

The chosen CASA system [12] computes pitch of each frame through summed autocorrelation function (SACF), and this pitch calculation process is composed of two stages: the global pitch computation stage and the refined pitch computation stage. Besides, a grouping strategy based on segments composed of T-F units coming from the same source is used. In this paper, we did the same thing in these two aspects.

\section{B. Unit Labeling}

Correctly labeling T-F units as target or interference is a key step in the source separation process. In the referred system, T-F units are first classified into resolved units and unresolved ones, meaning they respond to a single harmonic and multiple harmonics of the source respectively. Then a T-F unit is labeled target if it satisfies one of the following two formulas [12].

$$
\begin{aligned}
& \frac{A_{H}\left(c, m, \tau_{s}(m)\right)}{\max _{\tau \in \Gamma} A_{H}(c, m, \tau)}>\theta_{T} \\
& \frac{A_{E}\left(c, m, \tau_{s}(m)\right)}{\max _{\tau \in \Gamma} A_{E}(c, m, \tau)}>\theta_{A}
\end{aligned}
$$

As an indirect measurement of pitch similarity, it labels units as target ones only if left of the above formulas come with high ratios. Though effective in most cases, this method has the following two drawbacks.

(1) When the intrusion has a pitch which is multiples of the target voice, false labeling can also occurs.

(2) When the response or the response envelope of a unit is very flat, high ratios always appear, resulting in false acceptation.

The first problem could be solved by considering the instantaneous frequency of each T-F unit, but determination the instantaneous frequency itself is a hard task [4]. In this paper, we find that to involve the following two constraints into the labeling process is a simple but effective way to alleviate these two problems.

Constraint 1: If a T-F unit is unresolved and located above channel 60, it is included only if its ODACF is smaller than $\varphi_{A}$, and its ODEACF is larger than $\varphi_{E}$. This constraint can be represented by the following inequalities.

$$
\begin{gathered}
O D A C F(c, m)<\varphi_{A} \\
O D E A C F(c, m)>\varphi_{E}
\end{gathered}
$$

Constraint 2: If a T-F unit is resolved and located under channel 60, it is included only if its ODACF is larger than $\theta_{A}$. This criterion can be expressed by the following formula.

$$
O D A C F(c, m)>\theta_{A}
$$

Why do these constraints work? Speeches with higher pitch usually have larger ODACF and ODEACF as discussed in Section 2.3, so the multiple pitch faults can be alleviated by inequality (9) in Constraint 1 . The second problem can be partly solved by inequalities (10) and (11) for the unresolved and resolved units respectively, because signals with flat ACF tend to have very small ODACF, and flat EACF leads to small ODEACF.

So we label a unit 1 if it not only satisfies one of the above formulas in this section, but also satisfies both of the Constraints in this section. 
TABLE I

INTRUSIVE SOURCES.

\begin{tabular}{|c|c|}
\hline id & description \\
\hline $\mathrm{n} 0$ & $1 \mathrm{kHz}$ tone \\
\hline $\mathrm{n} 1$ & white noise \\
\hline $\mathrm{n} 2$ & series of brief noise bursts \\
\hline $\mathrm{n} 3$ & teaching laboratory noise \\
\hline $\mathrm{n} 4$ & new wave music \\
\hline $\mathrm{n} 5$ & FM signal (siren) \\
\hline $\mathrm{n} 6$ & telephone \\
\hline $\mathrm{n} 7$ & female TIMIT utterance \\
\hline $\mathrm{n} 8$ & male TIMIT utterance \\
\hline $\mathrm{n} 9$ & female utterance \\
\hline
\end{tabular}

\section{RESULTS AND ANALYSIS}

In this section, the CASA system improved by our method is evaluated systematically on Cooke set [6], which includes ten voiced sentences mixed respectively with ten different kinds of intrusions. The ten voiced utterances include five sentences, each spoken by two male speakers. The ten intrusions are listed in table 1.

To objectively evaluate the effect of segregation, we compute the signal to noise ratio (SNR) which is also a criterion used in the chosen system [12], and it is calculated by the following equation.

$$
S N R=10 \log _{10} \frac{\sum_{n} R^{2}(n)}{\sum_{n}[R(n)-S(n)]^{2}}
$$

Here, $R(n)$ is obtained by sending an original clean target utterance into the font-end processing part of the system and subsequently synthesizing it with an all-one mask. $S(n)$ is the real output of the improved system.

\section{A. The SNR Results}

Results of the original system and the modified system are shown in Table 2, as well as SNR of the original mixtures and the corresponding IBM (Ideal binary mask) results. As is often compared with, the results of an early system [7] is also listed in the table. The IBM results are obtained by passing the original clean utterances through the front-end part and then synthesizing them with ideal binary masks, which are determined in advance by local SNR of each T-F unit. Each value in Table 2 represents the average SNR for each intrusion mixed with 10 target utterances.

As we know, SNR results are not always consistent with human's subjective perception, and PESQ results are usually provided in other papers. For CASA, Wang proposed a computational goal [13] called Ideal Binary Mask (IBM), which is verified to be able to greatly increase the intelligibility of segregated sound. So in this paper, we also evaluated on this criterion by substituting the clean results $R(n)$ in equation (12) with the IBM results I(n). The revised SNR results are provided in Figure 4.
TABLE II

INTRUSIVE SOURCES.

\begin{tabular}{|c|c|c|c|c|c|}
\hline id & mixture & HW-04 & HW-06 & proposed & IBM \\
\hline n0 & -3.32 & 15.72 & 16.89 & 17.19 & 20.81 \\
\hline n1 & -4.14 & 7.69 & 7.65 & 7.91 & 9.13 \\
\hline n2 & 10.19 & 16.18 & 17.72 & 18.12 & 20.58 \\
\hline n3 & 4.21 & 8.31 & 8.94 & 8.92 & 9.90 \\
\hline n4 & 3.93 & 10.65 & 10.63 & 10.82 & 13.06 \\
\hline n5 & -5.89 & 14.45 & 15.58 & 15.62 & 17.80 \\
\hline n6 & 1.87 & 16.70 & 16.63 & 17.31 & 20.90 \\
\hline n7 & 6.58 & 11.89 & 11.63 & 12.49 & 15.51 \\
\hline n8 & 10.35 & 13.99 & 14.87 & 14.65 & 17.65 \\
\hline n9 & 0.67 & 3.94 & 5.35 & 5.77 & 11.15 \\
\hline ave & 2.44 & 11.95 & 12.59 & 12.90 & 15.56 \\
\hline
\end{tabular}

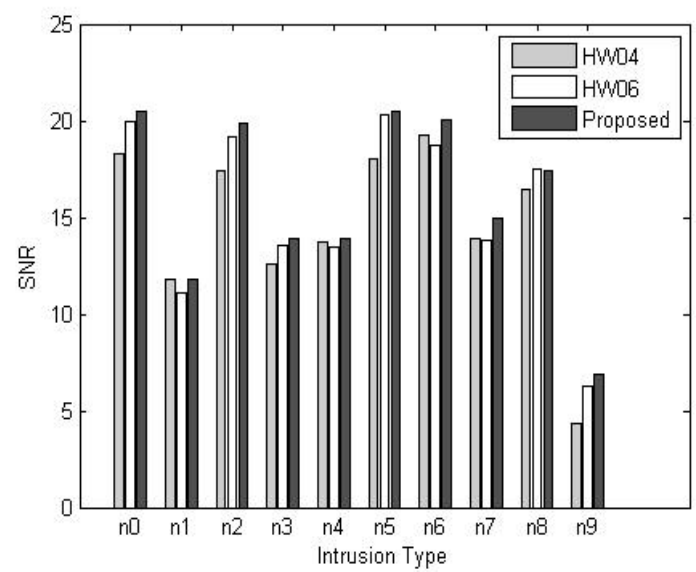

Fig. 4. SNR results against ideal binary masks.

\section{B. Analysis of The Results}

From the results in Figure 4, we can see that results obtained by the proposed system gain a consistent improvement except for $\mathrm{n} 8$. When IBM results is used as the goal, the new clues help the system gain an average enhancement of $0.6 \mathrm{~dB}$ compared with results of HW-06 [12].

It is reasonable to see these improvements. For $n 0$ and $n 2$, formula (11) successfully prevents most units of them in the low frequency regions from being labeled as target units. For $\mathrm{n} 1$, formula (10) plays a similar part. For n4, n6, n7 and n9, formula (9) and formula (10) both help a lot, thus unit labeling becomes more accurate for them. As a representative of these results, Figure 5 shows the spectrogram of the segregated v3 from its mixture with $\mathrm{n} 7$. As can be seen from the figure, most T-F units of $\mathrm{n} 7$ in the low frequency around $1 \mathrm{sec}$ are successful removed by the improved system. As Constraint 1 takes effect in the high channels, it may seems a little strange that T-F units of $\mathrm{n} 7$ in the low channels are also successfully removed. We think this is because more accurate unit labeling in the high channels helps the refined pitch computation process in determining more accurate target pitch.

As can be seen from Figure 4, there is no improvements 

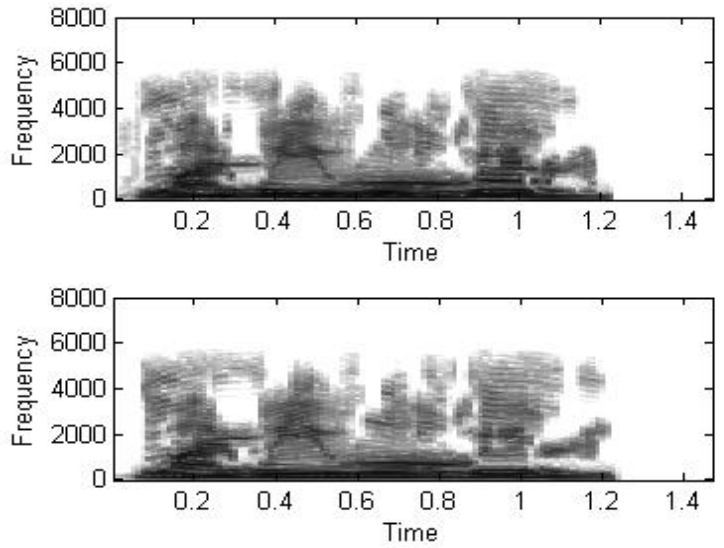

Fig. 5. Segregated v3 from its mixture with n7.

for the interference of $\mathrm{n} 8$. This happens because $\mathrm{n} 8$ is also a male utterance, whose oscillation pattern is very similar with the target speeches. That is to say, the features proposed in this paper (ODACF and ODEACF) can not help to segregate speeches from speakers of the same gender.

\section{SUMMARY AND COMCLUSIONS}

For data-driven computational auditory scene analysis (CASA) systems, effective clues for time frequency (T-F) unit labeling are of vital importance. In this paper, the spectral smoothness principle is modeled through the oscillation pattern represented by oscillation degree of autocorrelation function (ODACF) and of envelope autocorrelation (ODEACF) of each unit. We incorporate this clue in the unit labeling stage of a CASA system, which can also help in the refined pitch computation stage. Experiment results in Section 4 provide concrete supports for the conclusion that CASA systems can be obviously improved by our method except for utterances of the same gender.

\section{REFERENCES}

[1] S. Bregman, Auditory Scene Analysis, MA: MIT press, 1990.

[2] D.L. Wang and G.J. Brown, Ed., Computational auditory scene analysis: Principles, algorithms, and applications, New York: IEEE Press/Wiley, 2006.

[3] P. Li, Y. Guan, W. Liu and B. Xu, "Monaural speech separation based on computational auditory scene analysis and objective quality assessment of speech," in IEEE Trans. Audio, Speech, and Language Processing, Vol.14, 2006, pp. 2014-2023.

[4] G. Hu, Monaural Speech Organization and Segregation, Ph.D. dissertation, Biophysics Program, The Ohio State University, 2006.

[5] D. L. Wang, "Computational scene analysis," in Challenges for computational intelligence, W. Duch and J. Mandziuk (Eds.), Springer, Berlin, 2007, pp. 163-191.

[6] M. P. Cooke, Modeling Auditory Processing and Organization, U.K.: Cambridge University, 1993.

[7] G. Hu and D. L. Wang, "Monaural speech segregation based on pitch tracking and amplitude modulation," in IEEE Trans. Neural Netw., vol. 15, 2004, pp. 1135-1150.

[8] Xueliang Zhang, Wenju Liu, Peng Li and Bo Xu, "Monaural voiced speech segregation based on elaborate harmonic grouping strategy," in Proc. ICASSP 2009, pp. 4661-4664.
[9] G. Hu, D. L. Wang. "A tandem algorithm for pitch estimation and voiced speech segregation." in IEEE Trans. Audio, Speech, Lang. Process., vol. 18, 2010, pp. 2067-2079.

[10] A. P. Klapuri, "Multipitch estimation and sound separation by the spectral smoothness principle," in Proc. ICASSP, 2001, pp. 3381-3384.

[11] R. Meddis, "Simulation of auditory-neural transduction: further studies," in J. Acoust. Soc. Amer, vol 83, 1988, pp. 1056-1063.

[12] G. Hu and D.L. Wang, "An auditory scene analysis approach to monaural speech segregation," in Selected methods for acoustic echo and noise control, G. Schmidt and E. Haensler, Ed., Berlin: Springer, 2006.

[13] D. L. Wang, "On ideal binary mask as the computational goal of auditory scene analysis". In Speech Separation by Humans and Machines, P. Divenyi, Ed. Boston, MA: Kluwer, 2005. 